\title{
CONVERGENCE OF AVERAGES OF POINT TRANSFORMATIONS
}

\section{A. AKCOGLU ${ }^{1}$ AND A. DEL JUNCO}

Let $(X, \mathcal{F}, \mu)$ be a finite measure space. An invertible transformation of $X$ which is measure preserving in both directions is called an automorphism of $X$. Birkhoff's Ergodic Theorem states that if $\tau$ is an automorphism of $X$ then the sequence

$$
\frac{1}{n+1} \sum_{i=0}^{n} f\left(\tau^{i} x\right)
$$

converges a.e. for each $f \in L_{1}=L_{1}(X, \mathcal{F}, \mu)$. This raises the following question. What are the necessary conditions on the matrix $\left(a_{n i}\right)$ so that the sequence $f_{n}(x)=\Sigma_{i} a_{n i} f\left(\tau^{-i} x\right)$ converges a.e. for each $f \in L_{1}$ and for each automorphism $\tau$ ? The answer is not known. Spectral considerations would suggest, however, the following conjecture. If $\left(a_{n i}\right)$ is such that the sequence of functions $p_{n}(z)=\Sigma_{i} a_{n i} z^{-i}$ is uniformly bounded and pointwise convergent on the unit circle $|z|=1$, then $f_{n}$ converges a.e. In fact, recently an attempt has been made to prove this as a theorem [1]. In this note we would like to observe the following simple fact which shows that this conjecture is far from being correct.

If $r$ is a real number, let $[r]$ denote the greatest integer which is less than or equal to $r$. Define a matrix $\left(a_{n i}\right), n=1,2, \cdots$, as

$$
a_{n i}= \begin{cases}\frac{1}{[\sqrt{ } n]+1} & \text { if } n \leq i \leq[\sqrt{ } n]+n \\ 0 & \text { otherwise. }\end{cases}
$$

Then the $a_{n i}$ certainly satisfy the hypotheses of the conjecture. However, we have the following result.

Proposition. If $\tau$ is an ergodic automorphism of a probability space $(X, \mathcal{F}, \mu)$, then there is a set $E$ such that there is a set $B$ of positive measure on which $\Sigma_{i} a_{n i} X_{E}\left(\tau^{-i} x\right)$ fails to converge.

Received by the editors July 23, 1974.

AMS (MOS) subject classifications (1970). Primary 28A65, 47A35.

1 Research supported by NRC Grant A3974. 
Proof. Let $k_{m}=2^{m}$. By Rohlin's theorem (see e.g. [2, Theorem 8.1]) for each $m \geq 2$ there is a set $F_{m} \in \mathcal{F}$ such that $\tau^{i} F_{m}, 0 \leq i \leq k_{m}^{2}+k_{m}$, are mutually disjoint and

$$
\mu \bigcup_{i=0}^{k_{m}^{2}+k} \tau_{m}^{i} F_{m}>\frac{9}{10}
$$

Let

$$
E_{m}=\bigcup_{i=0}^{k} \tau_{m}^{i} F_{m}, \quad B_{m}=\bigcup_{i=2 k_{m}}^{k_{m}^{2}+k} \tau_{m}^{i} F_{m}
$$

Note that

$$
\begin{aligned}
& \mu\left(E_{m}\right)<\frac{1}{k_{m}}, \\
& \mu\left(B_{m}\right)>\left(1-\frac{2 k_{m}}{k_{m}^{2}+k_{m}}\right) \frac{9}{10}>\frac{1}{2} .
\end{aligned}
$$

Let $E=\bigcup_{m \geq 2} E_{m}$. Then (1) implies

$$
\mu(E) \leq \sum_{m \geq 2} \frac{1}{k_{m}}<\frac{1}{2} .
$$

$$
\text { If } x \in \tau^{n+k_{m}} F_{n}, k_{m} \leq n \leq k_{m}^{2} \text {, then }
$$

$$
\sum_{i} a_{n i} \chi_{\tau^{-i} E_{n}}(x)=1 .
$$

(4) implies that for each $x \in B_{m}$ there is an integer $n \geq k_{m}$ such that

$$
\sum_{i} a_{n i} \chi_{E}\left(\tau^{-i} x\right)=1
$$

Since $\mu\left(B_{m}\right)>1 / 2$, by Fatou's Lemma there is a set $B$ such that $\mu(B)>1 / 2$ and each $x \in B$ belongs to infinitely many of the $B_{m}$. Thus if $x \in B$, (5) holds for infinitely many integers $n$. However since $\tau$ is ergodic, if $\Sigma_{i} a_{n i} \chi_{E}\left(\tau^{-i} x\right)$ converges a.e., the limit function is equal to $\mu(E)$ a.e. Thus by (3) the convergence must fail.

\section{BIBLIOGRAPHY}

1. J. M. Belley, Invertible measure preserving transformations and pointwise convergence, Proc. Amer. Math. Soc. 43 (1974), 159-162.

2. M. Smorodinsky, Ergodic theory, entropy, Lecture Notes in Math., vol. 214, Springer-Verlag, New York and Berlin, 1971.

DEPARTMENT OF MATHEMATICS, UNIVERSITY OF TORONTO, TORONTO, ONTARIO, CANADA 